Pacific Journal of Mathematics

INVERSION OF CONDITIONAL WIENER INTEGRALS 


\title{
INVERSION OF CONDITIONAL WIENER INTEGRALS
}

\author{
Dedicated to Professor Robert H. Cameron \\ J. YEH
}

Given two Wiener measurable functionals $X$ and $Y$ on the Wiener space $C[0, t]$, of which the latter is Wiener integrable, the conditional Wiener integral of $Y$ given $X$ is defined as the conditional expectation $E^{w}(Y \mid X)$ given as a function on the value space of $X$. Several Fourier inversion formulae for retrieving the conditional Wiener integral $E^{w}(Y \mid X)$ in which $X[x]=x(t)$ for $x \in C[0, t]$ are derived. Examples of evaluation of $E^{w}(Y \mid X)$ are given. It is shown that the Kac-Feynman formula can be derived by applying an inversion formula to $E^{w}(Y \mid X)$ where

$$
Y[x]=\exp \left\{-\int_{0}^{t} V[x(s)] d s\right\} \text {. }
$$

1. Introduction. We have recently derived an inversion formula for conditional expectations. (See [4].) In the present paper we report on some inversion formulae for conditional Wiener integrals. Here the probability space is the Wiener measure space on the Wiener space $C[0, t]$ of the real valued continuous functions $x$ on $[0, t]$ with $x(0)=0$ for fixed $t \in(0, \infty)$. By a conditional Wiener integral we mean the conditional expectation $E^{w}(Y \mid X)$ of a real or complex valued Wiener integrable functional $Y$ conditioned by a Wiener measurable functional $X$ on $C[0, t]$ which is given as a function on the value space of $X$. We shall be concerned exclusively with $X$ given by $X[x]=$ $x(t)$ for $x \in C[0, t]$. Thus $E^{w}(Y \mid X)$ will be a real or complex valued function on $R^{1}$. A precise definition of conditional Wiener integral as well as a brief discussion of the Wiener measure space are given in §2. Three inversion formulae for conditional Wiener integrals are proved in §3: a (C. 1) summability type inversion formula (Theorem 1), a Lévy type inversion formula (Theorem 2) and an inversion formula under the assumption of the Lebseque integrability of $E^{w}\left[e^{i u x} Y\right]$, $u \in R^{1}$ (Theorem 3). Examples of evaluation of conditional Wiener integrals are given in $\S 4$. Below we relate conditional Wiener integrals to the Kac-Feynman formula.

Consider the Wiener integrable functional

$$
\exp \left\{-\int_{0}^{t} V[x(s)] d s\right\} \text { for } x \in C[0, t]
$$

where $V$ is a nonnegative continuous function on $R^{1}$. Under the 
additional condition that $V$ is bounded, M. Kac [2], showed that a real valued function $U$ on $R^{1} \times(0, \infty)$ defined by

$$
U(\xi, t)=\sum_{k=0}^{\infty}(-1)^{k} U_{k}(\xi, t) \quad \text { for } \quad(\xi, t) \in R^{1} \times(0, \infty)
$$

where the sequence $\left\{U_{k}, k=0,1,2, \cdots\right\}$ is defined inductively by

$$
\begin{gathered}
U_{0}(\xi, t)=\frac{1}{\sqrt{2 \pi t}} \exp \left\{-\frac{\xi^{2}}{2 t}\right\} \\
U_{k+1}(\xi, t)=\frac{1}{\sqrt{2 \pi}} \int_{0}^{t}\left\{\int_{-\infty}^{\infty} \frac{1}{\sqrt{t-s}} \exp \left\{-\frac{1}{2} \frac{(\xi-\eta)^{2}}{t-s}\right\} V(\eta) U_{k}(\eta, s) d \eta\right\} d s \\
\quad \text { for } k=0,1,2, \cdots
\end{gathered}
$$

satisfies the integral equation

$$
\begin{aligned}
U(\xi, t)= & \frac{1}{\sqrt{2 \pi t}} \exp \left\{-\frac{\xi^{2}}{2 t}\right\}-\frac{1}{\sqrt{2 \pi}} \int_{0}^{t} \\
& \times\left\{\int_{-\infty}^{\infty} \frac{1}{\sqrt{t-s}} \exp \left\{-\frac{1}{2} \frac{(\xi-\eta)^{2}}{t-s}\right\} V(\eta) U(\eta, s) d \eta\right\} d s
\end{aligned}
$$

for $(\xi, t) \in R^{1} \times(0, \infty)$ and the boundary condition

$$
\int_{a}^{b} U(\xi, t) d \xi=E^{w}\left[\exp \left\{-\int_{0}^{t} V[x(s)] d s\right\} ; a<x(t)<b\right]
$$

for any $(a, b) \subset R^{1}$. The boundedness condition on $V$ was then removed by the method of truncation. The integral equation (1.1) implies that $U$ satisfies the differential equation

$$
\frac{\partial U}{\partial t}=\frac{1}{2} \frac{\partial^{2} U}{\partial \xi^{2}}-V(\xi) U \text { for } \quad(\xi, t) \in R^{1} \times(0, \infty)
$$

and the boundary condition (1.2) implies that $U$ satisfies the initial condition

$$
\lim _{t \rightarrow 0} \int_{-\varepsilon}^{\varepsilon} U(\xi, t) d \xi=1 \text { for every } \varepsilon>0 .
$$

This result is summarized by the Kac-Feynman formula

$$
U(\xi, t)=E^{w}\left[\exp \left\{-\int_{0}^{t} V[x(s) d s\} \delta(x(t)-\xi)\right]\right.
$$

for the solution $U$ of the differential system (1.3) and (1.4). Now let $X_{t}$ and $Y_{t}$ be two real valued functionals on $C[0, t]$ defined by

$$
X_{t}[x]=x(t) \quad \text { and } \quad Y_{t}[x]=\exp \left\{-\int_{0}^{t} V[x(s)] d s\right\} \quad \text { for } \quad x \in C[0, t] .
$$


The fact that the function $U$ satisfies (1.2) indicates that it is related to the conditional Wiener integral $E^{w}\left(Y_{t} \mid X_{t}\right)$. Let us define a real valued function $U$ on $R^{1} \times(0, \infty)$ by

$$
\begin{aligned}
U(\xi, t)=E^{w}\left(Y_{t} \mid X_{t}\right)(\xi) \frac{1}{\sqrt{2 \pi t}} \exp \left\{-\frac{\xi^{2}}{2 t}\right\} & \\
& \text { for }(\xi, t) \in R^{1} \times(0, \infty) .
\end{aligned}
$$

In $\S 5$ we show that by applying Theorem 1 to an appropriately chosen version of $E^{w}\left(Y_{t} \mid X_{t}\right)$ we obtain the integral equation (1.1) for the function $U$ defined by (1.6). The fact our function $U$ satisfies (1.2) is obvious. This gives an alternate way of deriving the KacFeynman formula. ${ }^{1}$

2. Conditional Wiener integral. For a fixed $t \in(0, \infty)$ consider the Wiener measure space $\left(C[0, t], \mathfrak{W}^{*}, m_{w}\right)$ where $\mathfrak{W}$ is the algebra of subsets $W$ of $C[0, t]$ of the type

$$
W=\left\{x \in C[0, t] ;\left[x\left(s_{1}\right), \cdots, x\left(s_{n}\right)\right] \in B\right\}
$$

where $n$ is an arbitrary positive integer, $0=s_{0}<s_{1}<\cdots<s_{n} \leqq t$, and $B$ is an arbitrary member of the $\sigma$-algebra $\mathfrak{B}^{n}$ of the Borel sets in the $n$-dimensional Euclidean space $R^{n} ; m_{w}$ is a probability measure on the algebra $\mathfrak{W}$ defined for $W$ as in (2.1) by

$$
\begin{aligned}
m_{w}(W)= & \left\{(2 \pi)^{n} \prod_{j=1}^{n}\left(s_{j}-s_{j-1}\right)\right\}^{-1 / 2} \\
& \int_{B} \exp \left\{-\frac{1}{2} \sum_{j=1}^{n} \frac{\left(\xi_{j}-\xi_{j-1}\right)}{s_{j}-s_{j-1}}\right\} m_{L}(d \xi)
\end{aligned}
$$

where $\xi=\left(\xi_{1}, \cdots, \xi_{n}\right) \in R^{n}, \xi_{0}=0$ and $m_{L}$ is the Lebesque measure on $\left(R^{n}, \mathfrak{B}^{n}\right) ; \mathfrak{W}^{*}$ is the $\sigma$-algebra of Carathéodory measurable subsets of $C[0, t]$ with respect to the outer measure derived from the probability measure $m_{w}$ on the algebra $\mathfrak{B}$ which in particular contains the $\sigma$ algebra $\sigma(\mathfrak{W})$ generated by $\mathfrak{W}$.

A real valued functional $F$ on $C[0, t]$ is said to be Wiener measurable if it is $\mathfrak{W}^{*}$-measurable, i.e. if it is a measurable transformation of $\left(C[0, t], \mathfrak{S}^{*}\right)$ into $\left(R^{1}, \mathfrak{B}^{1}\right)$. For a Wiener measurable functional $F$ we write

$$
E^{w}[F] \text { for } \int_{c[0, t]} F[x] m_{w}(d x)
$$

1 The idea of deriving the Kac-Feynman formula by inversion goes back to M. D. Donsker. I wish to thank Professor Donsker for the conversations we had on this approach. 
whenever the integral, i.e. the Wiener integral, exists. We say that $F$ is Wiener integrable or $m_{w}$-integrable when the Wiener integral of $F$ exists and is finite. The Wiener measurability and Wiener integrability of a complex valued functional on $C[0, t]$ are defined in terms of its real and imaginary parts. An immediate consequence of the definition of the Wiener measure is that if $F$ is a real or complex valued functional on $C[0, t]$ of the type

$$
F[x]=f\left[x\left(s_{1}\right), \cdots, x\left(s_{n}\right)\right] \quad \text { for } x \in C[0, t]
$$

where $f$ is a real of complex valued Baire function on $R^{n}$ and $0<$ $s_{1}<\cdots<s_{n} \leqq t$ then $F$ is Wiener measurable and

$$
\begin{aligned}
E^{w}[F]= & \left\{(2 \pi)^{n} \prod_{j=1}^{n}\left(s_{j}-s_{j-1}\right)\right\}^{-1 / 2} \\
& \int_{R^{n}} f(\xi) \exp \left\{-\frac{1}{2} \sum_{j=1}^{n} \frac{\left(\xi_{j}-\xi_{j-1}\right)^{2}}{s_{j}-s_{j-1}}\right\} m_{L}(d \xi)
\end{aligned}
$$

in the sense that the existence of one side implies that of the other as well as the equality of the two.

In connection with the Wiener measure space let us remark that a real valued function $X$ on $[0, t] \times C[0, t]$ defined by

$$
X(s, x)=x(s) \text { for }(s, x) \in[0, t] \times C[0, t]
$$

is a Brownian motion process on the probability space $\left(C[0, t], \mathfrak{W}^{*}\right.$, $m_{w}$ ) and the domain $[0, t]$ in which the space of sample functions $X(\cdot, x), x \in C[0, t]$, coincides with the sample space $C[0, t]$ and thus every sample function is continuous. This last property implies in particular that the process is a measurable process. We shall refer to this realization of the Brownian motion process as the Wiener process on the domain $[0, t]$.

Definition. Let $X$ and $Z$ be real valued Wiener measurable functionals on the Wiener measure space $\left(C[0, t], \mathfrak{W}^{*}, m_{w}\right)$ and let $Z$ be Wiener integrable on $C[0, t]$. Let $P_{X}$ be the probability distribution of $X$, i.e. the probability measure on $\left(R^{1}, \mathfrak{B}^{1}\right)$ determined by $X$ by the definition

$$
P_{X}(B)=m_{w}\left(X^{-1}(B)\right) \text { for } \quad B \in \mathfrak{B}^{1} .
$$

The conditional Wiener integral of $Z$ given $X$, written $E^{w}(Z \mid X)$, is by definition the equivalence class of $\mathfrak{B}^{1}$-measurable and $P_{X}$-integrable functions $\psi$ on $R^{1}$ modulo null functions on $\left(R^{1}, \mathfrak{B}^{1}, P_{X}\right)$ such that 


$$
\int_{X^{-1}(B)} Z[x] m_{w}(d x)=\int_{B} \psi(\xi) P_{X}(d \xi) \quad \text { for } \quad B \in \mathfrak{B}^{1} .
$$

By the Radon-Nikodym theorem such a function $\psi$ exists and is determined up to a null function on $\left(R^{1}, \mathfrak{B}^{1}, P_{X}\right)$. We shall also use $E^{w}(Z \mid X)$ to mean a particular version, i.e. a particular representative of the equivalence class. Thus

$$
\int_{X^{-1}(B)} Z[x] m_{w}(d x)=\int_{B} E^{w}(Z \mid X)(\xi) P_{X}(d \xi) \text { for } B \in \mathfrak{B}^{1} .
$$

3. Inversion formulae of conditional Wiener integrals.

LeMma 1. Let $X$ and $Z$ be measurable transformations of $(C[0$, t], $\left.\mathfrak{B}^{*}\right)$ into $\left(R^{1}, \mathfrak{B}^{1}\right)$ with $E^{w}[|Z|]<\infty$. Let $g$ be a measurable transformation of $\left(R^{1}, \mathfrak{B}^{1}\right)$ into itself. Then

$$
E^{w}[(g \circ X) Z]=\int_{R^{1}} g(\xi) E^{w}(Z \mid X)(\xi) P_{X}(d \xi)
$$

in the sense that the existence of one side implies that of the other as well as the equality of the two.

Proof. This lemma is a particular case of Proposition 3 in [4].

Corollary. Let $X$ and $Z$ be as in Lemma 1. Assume that $P_{X} \ll m_{L}$ on $\left(R^{1}, \mathfrak{B}^{1}\right)$. For $\xi \in R^{1}$ and $a>0$ let $\chi_{\xi, a}$ be a function on $R^{1}$ defined by

$$
\chi_{\xi, a}(\eta)=\left\{\begin{array}{lll}
\frac{1}{2 a} & \text { for } & \eta \in[\xi-a, \xi+a] \\
0 & \text { for } & \eta \in[\xi-a, \xi+a]^{c} .
\end{array}\right.
$$

Then there exists a version of $E^{w}(Z \mid X)\left(d P_{X} / d m_{L}\right)$ such that

$$
E^{w}(Z \mid X)(\xi) \frac{d P_{X}}{d m_{L}}(\xi)=\lim _{a \rightarrow 0} E^{w}\left[\left(\chi_{\xi, a} \circ X\right) Z\right] \quad \text { for } \quad \xi \in R^{1} \text {. }
$$

Proof. Using $\chi_{\xi, a}$ in the place of $g$ in Lemma 1, we have

$$
\begin{aligned}
\lim _{a \rightarrow 0} E^{w}\left[\left(\chi_{\xi, a} \circ X\right) Z\right] \\
\quad=\lim _{a \rightarrow 0} \int_{R^{1}} \chi_{\xi, a}(\eta) E^{w}(Z \mid X)(\eta) \frac{d P_{X}}{d m_{L}}(\eta) m_{L}(d \eta) .
\end{aligned}
$$

It is well known that if $f \in L_{1 \mathrm{oc}}^{1}\left(R^{1}, \mathfrak{B}^{1}, m_{L}\right)$ then

$$
\lim _{a \rightarrow 0} \int_{R^{1}} \chi_{\xi, a}(\eta) f(\eta) m_{L}(d \eta)=f(\xi) \text { for } m_{L^{-}} \text {a.e. } \xi \in R^{1} .
$$


From this follows our corollary.

Lemma 2. Let $X$ and $Y$ be measurable transformations of $(C[0$, t], $\left.\mathfrak{B}^{*}\right)$ into $\left(R^{1}, \mathfrak{B}^{1}\right)$ with $E^{w}[|Y|]<\infty$. Then

$$
E^{w}\left[e^{i u X} Y\right]=\int_{R^{1}} e^{i u \xi} E^{w}(Y \mid X)(\xi) P_{X}(d \xi) \quad \text { for } \quad u \in R^{1} .
$$

Proof. This lemma is the equality (3.19) in [4] adapted to our probability space $\left(C[0, t], \mathfrak{W}^{*} m_{w}\right)$.

Theorem 1. Let $X$ and $Y$ be measurable transformations of $\left(C[0, t], \mathfrak{B}^{*}\right)$ into $\left(R^{1}, \mathfrak{B}^{1}\right)$ with $E^{w}[|Y|]<\infty$. Assume that $P_{X} \ll m_{L}$ on $\left(R^{1}, \mathfrak{B}^{1}\right)$. Then there exists a version of $E^{w}(Y \mid X)\left(d P_{X} / d m_{L}\right)$ such that

$$
\begin{aligned}
& E^{w}(Y \mid X)(\xi) \frac{d P_{X}}{d m_{L}}(\xi) \\
& \quad=\lim _{a \rightarrow \infty} \frac{1}{2 \pi} \int_{(-a, a)}\left(1-\frac{|u|}{a}\right) e^{-i u \xi} E^{w}\left[e^{i u X} Y\right] m_{L}(d u) \text { for } \xi \in R^{1} .
\end{aligned}
$$

Proof. By Lemma 2

$$
E^{w}\left[e^{i u X} Y\right]=\int_{R^{1}} e^{i u \xi} E^{w}(Y \mid X)(\xi) \frac{d P_{X}}{d m_{L}}(\xi) m_{L}(d \xi) \quad \text { for } \quad u \in R^{1} .
$$

Thus our $E^{w}\left[e^{i u x} Y\right], u \in R^{1}$, is the Fourier transform of the $m_{L^{-}}$ integrable function $E^{w}(Y \mid X)(\xi)\left(d P_{X} / d m_{L}(\xi), \xi \in R^{1}\right.$. According to a well known $(\mathrm{C}, 1)$ summability type inversion formula for Fourier transforms, if $f$ is a $m_{L}$-integrable function on $R^{1}$ and $\hat{f}$ is its Fourier transform then

$$
f(\xi)=\lim _{a \rightarrow \infty} \frac{1}{2 \pi} \int_{(-a, a)}\left(1-\frac{|u|}{a}\right) e^{-i u \xi} \hat{f}(u) m_{L}(d u) \quad \text { for } \quad m_{L} \text {-a.e. } \xi \in R^{1}
$$

(see for instance [1]). From this follows our theorem.

Theorem 2. Let $X$ and $Y$ be as in Theorem 1. For $a, b \in R^{1}$ such that $a<b$ let $\tilde{\chi}_{a, b}$ be a function on $R^{1}$ defined by

$$
\tilde{\chi}_{a, b}(\eta)= \begin{cases}1 & \text { for } \eta \in(a, b) \\ 0 & \text { for } \eta \in[a, b]^{c} \\ \frac{1}{2} & \text { for } \eta=a \text { and for } \eta=b .\end{cases}
$$

Then 


$$
\begin{aligned}
& \int_{R^{1}} \tilde{\chi}_{a, b}(\xi) E^{w}(Y \mid X)(\xi) \frac{d P_{X}}{d m_{L}}(\xi) m_{L}(d \xi) \\
& \quad=\lim _{h \rightarrow \infty} \frac{1}{2 \pi} \int_{(-h, h)} \frac{e^{-i b u}-e^{-i a u}}{-i u} E^{w}\left[e^{i u X} Y\right] m_{L}(d u)
\end{aligned}
$$

Proof. As an immediate consequence of the Lévy inversion formula, if $\Phi$ is a finite signed measure on $\left(R^{1}, B^{1}\right)$ and if $\phi$ is the characteristic function $\Phi$, i.e.

$$
\varphi(u)=\int_{R^{1}} e^{i u \xi} \Phi(d \xi) \quad \text { for } \quad u \in R_{\downarrow}
$$

then we have

$$
\int_{R^{1}} \tilde{\chi}_{a, b}(\xi) \Phi(d \xi)=\lim _{h \rightarrow \infty} \frac{1}{2 \pi} \int_{(-h, h)} \frac{e^{-i b u}-e^{-i a u}}{-i u} \varphi(u) m_{L}(d u) .
$$

From this and from (3.1) follows our theorem.

THEOREM 3. Let $X$ and $Y$ be as in Theorem 1. Assume further that $E^{w}\left[e^{i u X} Y\right]$ is a $m_{L}$-integrable function of $u$ on $R^{1}$. Then $a$ version of $E^{w}(Y \mid X)\left(d P_{X} / d m_{L}\right)$ is given by

$$
E^{w}(Y \mid X)(\xi) \frac{d P_{X}}{d m_{L}}(\xi)=\frac{1}{2 \pi} \int_{R^{1}} e^{-i u \xi} E^{w}\left[e^{i u X} Y\right] m_{L}(d u) \quad \text { for } \quad \xi \in R^{1} .
$$

Proof. This theorem is a particular case of Theorem 2 in [4].

4. Examples of evaluation of conditional Wiener integrals.

EXAMPLE $1 .^{2}$ For each $x \in C[0, t]$ consider the average value of $x$ over the time interval $[0, t]$, i.e. $1 / t \int_{0}^{t} x(s) d s$. Let us find the conditional expectation of this average value given the condition that the final value of $x, x(t)$, is equal to $\xi \in R^{1}$. Thus we are to find $E^{w}(Z \mid X)(\xi), \xi \in R^{1}$, for

$$
Z[x]=\frac{1}{t} \int_{0}^{t} x(s) d s \text { and } X[x]=x(t) \text { for } x \in C[0, t] .
$$

According to Corollary to Lemma 1 , a version of $E^{w}(Z \mid X)\left(d P_{X} / d m_{L}\right)$ is given by

$$
E^{w}(Z \mid X)(\xi) \frac{d P_{X}}{d m_{L}}(\xi)=\lim _{a \rightarrow 0} E^{w}\left[\left(\chi_{\xi, a} o X\right) Z\right] \text { for } \xi \in R^{1} .
$$

${ }^{2}$ According to Professor E. O. Thorp, this problem arises in the probabilistic study of the fluctuation of the price of a commodity as a Brownian motion process. The problem was to find the expectation of the time average of the price when the price at some fixed time point in the future is known for some reason. 
With our $Z$ and $X$ we have

$$
E^{w}\left[\left(\chi_{\xi, a} \circ X\right) Z\right]=E^{w}\left[\chi_{\xi, a}[x(t)] \frac{1}{t} \int_{[0, t]} x(s) m_{L}(d s)\right] .
$$

An interchange of the order of the two integrations on the right side of (4.2), one with respect to the Lebesque measure and the other with respect to the Wiener measure, can be justified as follows. Recall first that the Wiener process is a measurable process, i.e. $x(s)$ for $(s, x) \in[0, t] \times C[0, t]$ is Lebesque $\times$ Wiener measurable. To apply the Fubini theorem observe that

$$
\left|\chi_{s, a}[x(t)] x(s)\right| \leqq \frac{1}{2 a}|x(s)| \quad \text { for } \quad(s, x) \in[0, t] \times C[0, t]
$$

and by (2.4)

$$
\begin{gathered}
\int_{[0, t]} E^{w}\left[\frac{1}{2 a}|x(s)|\right] m_{L}(d s)=\frac{1}{2 a} \int_{[0, t]}\left\{\frac{1}{\sqrt{2 \pi s}} \int_{R^{1}}|\eta| e^{-\eta^{2 / 2 s}} m_{L}(d \eta)\right\} m_{L}(d s) \\
=\frac{1}{2 a} \sqrt{\frac{2}{\pi}} \int_{[0, t]} \sqrt{s} m_{L}(d s)=\frac{1}{3 a} \sqrt{\frac{2}{\pi}} t^{3 / 2}<\infty .
\end{gathered}
$$

Thus an interchange of the two integrals on the right side of (4.2) is justified and consequently

$$
\begin{aligned}
E^{w}\left[\left(\chi_{\xi, a} \circ X\right) Z\right]=\frac{1}{t} \int_{[0, t]} E^{w}\left[\chi_{\xi, a}[x(t)] x(s)\right] m_{L}(d s) \\
\begin{aligned}
(4.3)= & \frac{1}{2 a t} \int_{[0, t]}\left\{(2 \pi)^{2} s(t-s)\right\}^{-1 / 2} \\
& \times\left[\int_{R^{1} \times[\xi-a, \xi+a]} \eta \exp \left\{-\frac{1}{2} \frac{\eta^{2}}{s}-\frac{1}{2} \frac{(\zeta-\eta)^{2}}{t-s}\right\} m_{L}(d(\eta, \zeta))\right] m_{L}(d s)
\end{aligned}
\end{aligned}
$$

by (2.4). Since

$$
\frac{\eta^{2}}{s}+\frac{(\zeta-\eta)^{2}}{t-s}=\frac{t}{s(t-s)}\left(\eta-\frac{s}{t} \zeta\right)^{2}+\frac{\zeta^{2}}{t}
$$

and thus

$$
\begin{aligned}
& \eta \exp \left\{-\frac{1}{2} \frac{\eta^{2}}{s}-\frac{1}{2} \frac{(\zeta-\eta)^{2}}{t-s}\right\} \\
& =\left\{\left(\eta-\frac{s}{t} \zeta\right)+\frac{s}{t} \zeta\right\} \exp \left\{-\frac{1}{2} \frac{t}{s(t-s)}\left(\eta-\frac{s}{t} \zeta\right)^{2}-\frac{1}{2} \frac{\zeta^{2}}{t}\right\}
\end{aligned}
$$

the integral with respect to $m_{L}(d(\eta, \zeta))$ over $R^{1} \times[\xi-a, \xi+a]$ in (4.3) can be reduced by integrating with respect to $m_{L}(d \eta)$ over $R^{1}$ and using 


$$
\begin{aligned}
& \frac{1}{\sqrt{2 \pi v}} \int_{R^{1}} y^{p} e^{-y^{2} / 2 v} m_{L}(d y)=1,0, v \text { for } p=0,1,2 \\
& \text { respectively, with } v>0
\end{aligned}
$$

to

$$
\sqrt{\frac{2 s^{3}(t-s) \pi}{t^{3}}} \int_{[\xi-a, \xi+a]} \zeta \exp \left\{-\frac{1}{2} \frac{\zeta^{2}}{t}\right\} m_{L}(d \zeta)
$$

Using this in (4.3) we have

$$
\begin{aligned}
& \left.E^{w}\left[\chi_{\xi, a} \circ X\right) Z\right] \\
& \quad=\frac{1}{\sqrt{2 \pi t} 2 a t^{2}}\left\{\int_{[0, t]} s m_{L}(d s)\right\}\left\{\int_{[\xi-a, \xi+a]} \zeta \exp \left\{-\frac{1}{2} \frac{\zeta^{2}}{t}\right\} m_{L}(d \zeta)\right. \\
& \quad=\frac{1}{2}\left[\frac{1}{2 a} \int_{[\xi-a, \xi+a]} \zeta \frac{1}{\sqrt{2 \pi t}} \exp \left\{-\frac{1}{2} \frac{\zeta^{2}}{t}\right\} m_{L}(d \zeta)\right] .
\end{aligned}
$$

From (4.1) and (4.6) we have

$$
E^{w}(Z \mid X)(\xi) \frac{d P_{X}}{d m_{L}}(\xi)=\frac{1}{2} \xi \frac{1}{\sqrt{2 \pi t}} \exp \left\{-\frac{1}{2} \frac{\xi^{2}}{t}\right\}
$$

and thus

$$
E^{w}(Z \mid X)(\xi)=\frac{1}{2} \xi
$$

ExAMPLe 2. Let us find $E^{w}(Z \mid X)(\xi), \xi \in R^{1}$, when

$$
Z[x]=\int_{0}^{t}[x(s)]^{2} d s \text { and } X[x]=x(t) \text { for } x \in C[0, t] .
$$

To apply Corollary of Lemma 1 we proceed as in Example 1 to obtain

$$
\begin{aligned}
E^{w} & {\left[\left(\chi_{\xi, a} \circ X\right) Z\right]=E^{w}\left[\chi_{\xi, a}[x(t)] \int_{0}^{t}[x(s)]^{2} d s\right] } \\
= & \int_{[0, t]} E^{w}\left[\chi_{\xi, a}[x(t)]\{x(s)\}^{2}\right] m_{L}(d s) \\
= & \frac{1}{2 a} \int_{[0, t]}\left\{(2 \pi)^{2} s(t-s)\right\}^{-1 / 2} \\
& \times\left[\int_{R^{1} \times[\xi-a, \xi+a]} \eta^{2} \exp \left\{-\frac{1}{2} \frac{\eta^{2}}{s}-\frac{1}{2} \frac{(\zeta-\eta)^{2}}{t-s}\right\} m_{L}(d(\eta, \zeta))\right] m_{L}(d s) .
\end{aligned}
$$

By means of (4.4) we have

$$
\begin{aligned}
\eta^{2} \exp & \left\{-\frac{1}{2} \frac{\eta^{2}}{s}-\frac{1}{2} \frac{(\zeta-\eta)^{2}}{t-s}\right\} \\
= & \left\{\left(\eta-\frac{s}{t} \zeta\right)^{2}+2 \frac{s}{t} \zeta\left(\eta-\frac{s}{t} \zeta\right)+\left(\frac{s}{t} \zeta\right)^{2}\right\}
\end{aligned}
$$




$$
\times \exp \left\{-\frac{1}{2} \frac{t}{s(t-s)}\left(\eta-\frac{s}{t} \zeta\right)^{2}-\frac{1}{2} \frac{\zeta^{2}}{t}\right\} .
$$

Reducing the integral with respect to $m_{L}(d(\eta, \zeta))$ over $R^{1} \times[\xi-a$, $\xi+a$ ] by integrating with respect to $m_{L}(d \eta)$ over $R^{1}$ using (4.5) we obtain

$$
\int_{[\xi-a, \xi+a]} \sqrt{\frac{2 \pi s(t-s)}{t}}\left\{\frac{s(t-s)}{t}+\left(\frac{s}{t} \zeta\right)^{2}\right\} e^{-\zeta^{2 / 2 t}} m_{L}(d \zeta)
$$

Using this in (4.7) we have

$$
\begin{aligned}
E^{w}\left[\left(\chi_{\xi, a} \circ X\right) Z\right] & \\
& =\frac{1}{\sqrt{2 \pi t} 2 a} \int_{[\xi-a, \xi+a]}\left[\int_{[0, t]}\left(s-\frac{s^{2}}{t}+\frac{s^{2}}{t^{2}} \zeta^{2}\right) m_{L}(d s)\right] e^{-\zeta^{2} / 2 t} m_{L}(d \zeta) \\
& =\frac{1}{2 a} \int_{[\xi-a, \xi+a]}\left(\frac{t^{2}}{6}+\frac{t}{3} \zeta^{2}\right) \frac{1}{\sqrt{2 \pi t}} e^{-\zeta^{2} / 2 t} m_{L}(d \zeta) .
\end{aligned}
$$

Thus

$$
\begin{gathered}
E^{w}(Z \mid X)(\xi) \frac{d P_{X}}{d m_{L}}(\xi)=\lim _{a \rightarrow 0} E^{w}\left[\left(\chi_{\zeta, a} \circ X\right) Z\right] \\
=\left(\frac{t^{2}}{6}+\frac{t}{3} \xi^{2}\right) \frac{1}{\sqrt{2 \pi t}} e^{-\xi^{2} / 2 t}
\end{gathered}
$$

and

$$
E^{w}(Z \mid X)(\xi)=\frac{t^{2}}{6}+\frac{t}{3} \xi^{2}
$$

It is of interest to note that from (2.5) and (4.8)

$$
\begin{aligned}
E^{w}[Z] & =\int_{X^{-1}\left(R^{1)}\right)} Z[x] m_{w}(d x)=\int_{R^{1}} E^{w}(Z \mid X)(\xi) P_{X}(d \xi) \\
& =\frac{1}{\sqrt{2 \pi t}} \int_{R^{1}}\left(\frac{t^{2}}{6}+\frac{t}{3} \xi^{2}\right) e^{-\xi^{2 / 2 t}} m_{L}(d \xi)=\frac{t^{2}}{2}
\end{aligned}
$$

as can be obtained by a direct computation of $E^{w}[Z]$.

As an example of application of an inversion formula in evaluating a condition Wiener integral, consider the Wiener measure space $\left(C[0, t], \mathfrak{W}^{*}, m_{w}\right)$ with fixed $t \in(0, \infty)$. Let $\left\{\varphi_{1}, \cdots, \varphi_{n}\right\}$ be an orthogonal system in the real Hilbert space $L^{2}([0, t])$ with

$$
\left\|\varphi_{j}\right\|=\sqrt{\int_{[0, t]}\left\{\varphi_{j}(s)\right\}^{2} m_{L}(d s)}>0 \quad \text { for } \quad j=1.2, \cdots, n
$$

such that $\varphi_{j}$ has a representative function which is of bounded variation 
on $[0, t]$ for $j=1,2, \cdots, n$. Let $F$ be a functional on $C[0, t]$ defined by

$$
F[x] f\left[\int_{0}^{t} \varphi_{1}(s) d x(s), \cdots, \int_{0}^{t} \varphi_{n}(s) d x(s)\right] \text { for } \quad x \in C[0, t]
$$

where $f$ is a complex valued function on $R^{n}$ whose real and imaginary parts are Baire functions. By a slight extension of a well known theorem by R. E. A. C. Paley, N. Wiener, and A. Zygmund [3] $F$ is Wiener measurable and

(4.10) $\quad E^{w}[F]=\left\{(2 \pi)^{n} \prod_{j=1}^{n}\left\|\varphi_{j}\right\|^{2}\right\}^{-1 / 2} \int_{R^{n}} f(\eta) \exp \left\{-\sum_{j=1}^{n} \frac{1}{2} \frac{\eta_{j}^{2}}{\left\|\varphi_{j}\right\|^{2}}\right\} m_{L}(d \eta)$

with $\eta=\left(\eta_{1}, \cdots, \eta_{n}\right) \in R^{n}$, in the sense that the existence of one side implies that of the other and the equality of the two.

THEOREM 4. Let $X$ and $Y$ be two functionals on $C[0, t]$ for fixed $t \in(0, \infty)$ defined by

$$
X[x]=x(t)
$$

and

$$
Y[x]=g\left[\int_{0}^{t} \varphi_{1}(s) d x(s), \cdots, \int_{0}^{t} \varphi_{n}(s) d x(s)\right] \text { for } \quad x \in C[0, t]
$$

where $\left\{\varphi_{1}, \cdots, \varphi_{n}, 1\right\}$ is an orthogonal system in the real Hilbert space $L^{2}([0, t]),\left\|\varphi_{j}\right\|>0, \varphi_{j}$ has a representative function which is of bounded variation on $[0, t]$ for $j=1,2, \cdots, n$ and $g$ is a Baire function on $R^{n}$ such that

(4.12) $\quad A=\left\{(2 \pi)^{n} \prod_{j=1}^{n}\left\|\varphi_{j}\right\|^{2}\right\}^{-1 / 2} \int_{R^{n}} g(\eta) \exp \left\{-\sum_{j=1}^{n} \frac{1}{2} \frac{\eta_{j}^{2}}{\left\|\varphi_{j}\right\|^{2}}\right\} m_{L}(d \eta)<\infty$ with $\eta=\left(\eta_{1}, \cdots, \eta_{n}\right) \in R^{n}$. Then $Y$ is Wiener integrable on $C[0, t]$ and

$$
E^{w}[Y]=A
$$

Also there exists a version of $E^{w}(Y \mid X)$ such that

$$
E^{w}(Y \mid X)(\xi)=A \quad \text { for } \xi \in R^{1} \text {. }
$$

Proof. By applying (4.10) to (4.11) we have (4.13). To prove (4.14) note that

$$
E^{w}\left[e^{i u x} Y\right]=E^{w}\left[\exp \left\{i u \int_{0}^{t} d x(s)\right\} g\left[\int_{0}^{t} \varphi_{1}(s) d x(s), \cdots, \int_{0}^{t} \varphi_{n}(s) d x(s)\right]\right] .
$$

Applying (4.10) to the function 


$$
f\left(\eta_{1}, \cdots, \eta_{n}, \xi\right)=e^{i u \xi} g\left(\eta_{1}, \cdots, \eta_{n}\right) \text { for }\left(\eta_{1}, \cdots, \eta_{n}, \xi\right) \in R^{n+1}
$$

we have

$$
E^{w}\left[e^{i u X} Y\right]=A \frac{1}{\sqrt{2 \pi t}} \int_{R^{1}} e^{i u \xi} e^{-\xi^{2} / 2 t} m_{L}(d t)=A e^{-u^{2} t / 2}
$$

since

$$
\begin{aligned}
& \int_{R^{1}} e^{-\left(a \xi^{2}+b \xi\right)} m_{L}(d \xi)=\sqrt{\frac{\pi}{a}} e^{b^{2 / 4 a}} \text { for } a>0 \text { and real or } \\
& \text { imaginary } b .
\end{aligned}
$$

We have just shown that $E^{w}\left[e^{i u X} Y\right]$ is a $m_{L}$-integrable function of $u$ on $R^{1}$. Thus by Theorem 3 there exists a version of $E^{w}(Y \mid X) d P_{X} / d m_{L}$ such that

$$
E^{w}(Y \mid X)(\xi) \frac{d P_{X}}{d m_{L}}(\xi)=\frac{A}{2 \pi} \int_{R^{1}} e^{-i u \xi} e^{-u^{2} t / 2} m_{L}(d u)=\frac{A}{2 \pi t} e^{-\xi^{2} / 2 t}
$$

and

$$
E^{w}(Y \mid X)(\xi)=A \quad \text { for } \xi \in R^{1}
$$

5. The conditional Wiener integral of $\exp \left\{-\int_{0}^{t} V[x(s)] d s\right\}$ given $x(t)$.

TheOREM 5. For $t \in(0, \infty)$ let

$$
\text { (5.1) } X_{t}[x]=x(t) \text { and } Y_{t}[x]=\exp \left\{-\int_{0}^{t} V[x(s)] d s\right\} \text { for } x \in C[0, t]
$$

where $V$ is a nonnegative continuous function on $R^{1}$ satisfying the condition

$$
\int_{R^{1}} V(\xi) e^{-\xi^{2 / 2 t}} m_{L}(d \xi)<\infty \quad \text { for every } \quad t \in(0, \infty) .
$$

There exists a version of $E^{w}\left(Y_{t} \mid X_{t}\right)$ such that the function $U$ on $R^{1} \times(0, \infty)$ defined by

$$
U(\xi, t)=E^{w}\left(Y_{t} \mid X_{t}\right)(\xi) \frac{1}{\sqrt{2 \pi t}} e^{-\xi^{2} / 2 t}
$$

satisfies the integral equation

$$
\begin{aligned}
U(\xi, t) & =\frac{1}{\sqrt{2 \pi t}} e^{-\xi^{2} / 2 t} \\
& -\int_{[0, t]}\left\{\int_{R^{1}} V(\eta) \frac{1}{\sqrt{2 \pi(t-s)}} e^{\left.-1 / 2(\xi-r)^{2} / t-s\right)} U(\eta, s) m_{L}(d \eta)\right\} m_{L}(d s)
\end{aligned}
$$


for $(\xi, t) \in R^{1} \times(0, \infty)$.

REMARK. From the continuity of $V$ on $R^{1}$ and the continuity of $x$ on $[0, t], \int_{0}^{t} V[x(s)] d s$ can be given as the limit of a sequence of Riemann sums corresponding to a sequence of partitions on $[0, t]$ which does not depend on $x$. From this follows the Wiener measurability of $Y_{t}$ on $C[0, t]$. Its Wiener integrability is obvious since it is bounded by 1 .

The condition (5.2) on $V$ is satisfied if for instance $V$ satisfies the order of growth condition

$$
V(\xi)=O\left(e^{\xi^{2-\delta}}\right) \text { as } \xi \longrightarrow \pm \infty
$$

for some $\delta \in(0,2)$. Note also that under (5.2), if we define

$$
\varphi(t)=\frac{1}{\sqrt{2 \pi t}} \int_{R^{1}} V(\xi) e^{-\xi^{2} / 2 t} m_{L}(d \xi) \quad \text { for } \quad t \in(0, \infty)
$$

then $\varphi$ is a nonnegative continuous function on $(0, \infty)$ and furthermore $\lim _{t \rightarrow 0} \varphi(t)=V(0)$. Let us define

$$
\varphi(0)=\lim _{t \rightarrow 0} \varphi(t)
$$

so that $\varphi$ is continuous on $[0, \infty)$.

Proof of Theorem 5. According to Theorem 1, there exists a version of $E^{w}\left(Y_{t} \mid X_{t}\right)$ such that our $U$ defined by (5.3) can be given as

$$
\begin{array}{r}
U(\xi, t)=\lim _{a \rightarrow \infty} \frac{1}{2 \pi} \int_{(-a, a)}\left(1-\frac{|u|}{a}\right) e^{-i u \xi} E^{W}\left[e^{-i u X_{t}} Y_{t}\right] m_{L}(d u) \\
\text { for }(\xi, t) \in R^{1} \times(0, \infty) .
\end{array}
$$

Since

$$
\frac{d}{d s} \exp \left\{-\int_{0}^{s} V[x(s)] d r\right\}=-\exp \left\{-\int_{0}^{s} V[x(r)] d r\right\} V[x(s)]
$$

we have by integrating with respect to $s$ on $[0, t]$

$$
\exp \left\{-\int_{0}^{t} V[x(s)] d s\right\}-1=-\int_{0}^{t} V[x(s)] \exp \left\{-\int_{0}^{s} V[x(r)] d r\right\} d s
$$

Applying (5.7) to $Y_{t}[x]$ as defined by (5.1) we have

$$
Y_{t}[x]=1-\int_{0}^{t} V[x(s)] \exp \left\{-\int_{0}^{s} V[x(r)] d r\right\} d s .
$$

Substituting (5.8) in (5.6) we have 


$$
U(\xi, t)=I_{1}(\xi, t)-I_{2}(\xi, t)
$$

where

(5.10) $\quad I_{1}(\xi, t)=\lim _{a \rightarrow \infty} \frac{1}{2 \pi} \int_{(-a, a)}\left(1-\frac{|u|}{a}\right) e^{-i u \xi} E^{w}\left[e^{i u x(t)}\right] m_{L}(d u)$

and

$$
I_{2}(\xi, t)=\lim _{a \rightarrow \infty} \frac{1}{2 \pi} \int_{(-a, a)}\left(1-\frac{|u|}{a}\right) e^{-i u \xi} J(t) m_{L}(d u)
$$

with

(5.12) $J(t)=E^{w}\left[e^{i u x(t)} \int_{[0, t]} V[x(s)] \exp \left\{-\int_{0}^{s} V[x(r)] d r\right\} m_{L}(d s)\right]$.

To evaluate $I_{1}$, note that by (2.4) and (4.15) we have

$$
E^{w}\left[e^{i u x(t)}\right]=\frac{1}{\sqrt{2 \pi t}} \int_{R^{1}} e^{i u \eta} e^{-\eta^{2} / 2 t} m_{L}(d \eta)=e^{-u^{2} t} / 2
$$

and thus

$$
\begin{aligned}
I_{1}(\xi, t)= & \lim _{a \rightarrow \infty}\left\{\frac{1}{2 \pi} \int_{(-a, a)} e^{-i u \xi} e^{-u^{2} t / 2} m_{L}(d u)-\frac{1}{2 \pi}\right. \\
& \left.\int_{(-a, a)} \frac{|u|}{a} e^{-i u \xi} e^{-u^{2} t / 2} m_{L}(d u)\right\} \\
= & \frac{1}{2 \pi} \int_{R^{1}} e^{-i u \xi} e^{-u^{2} t / 2} m_{L}(d u)-\frac{1}{2 \pi}\left(\lim _{u \rightarrow \infty}+\lim _{u \rightarrow-\infty}\right)\left(\frac{|u|}{a} e^{-i u \xi} e^{-u^{2} t / 2}\right) \\
= & \frac{1}{\sqrt{2 \pi t}} e^{-\xi^{2} / 2 t} .
\end{aligned}
$$

To interchange the order of the Wiener integral and the integral with respect to $m_{L}(d s)$ on $[0, t]$ in $(5.12)$, note that

$$
\begin{aligned}
&\left|e^{i u x(t)} V[x(s)] \exp \left\{-\int_{0}^{s} V[x(r)] d r\right\}\right| \leqq V[x(s)] \\
& \text { for } \quad(s, x) \in[0, t] \times C[0, t]
\end{aligned}
$$

and that by (2.4), (4.15), (5.5) and the continuity of $\varphi$ on $[0, \infty)$

$$
\begin{aligned}
\int_{[0, t]} E^{w}[V[x(s)]] m_{L}(d s) & =\int_{[0, t]} \frac{1}{\sqrt{2 \pi s}}\left\{\int_{R^{1}} V(\zeta) e^{-\zeta^{2 / 2 s}} m_{L}(d \zeta)\right\} m_{L}(d s) \\
= & \int_{[0, t]} \varphi(s) m_{L}(d s)<\infty
\end{aligned}
$$

so that the Fubini theorem is applicable and thus 


$$
J(t)=\int_{[0, t]} E^{w[}\left[e^{i u x(t)} V[x(s)] \exp \left\{-\int_{0}^{s} V[x(r)] d r\right\}\right] m_{L}(d s) .
$$

Let us write

$$
e^{i u x(t)}=e^{i u\{x(t)-x(s)\}} e^{i u x(s)} .
$$

By the fact that $\{x(t)-x(s), x(r)\}$ is an independent system of random variables on $\left(C[0, t], \mathfrak{W}^{*}, m_{w}\right)$ for every $r \in(0, s]$ and by

$$
E^{w}\left[e^{i u[x(t)-x(s)]}\right]=e^{-\left(u^{2} / 2\right)(t-s)}
$$

which follows from (2.4) and (4.15), we have from (5.14)

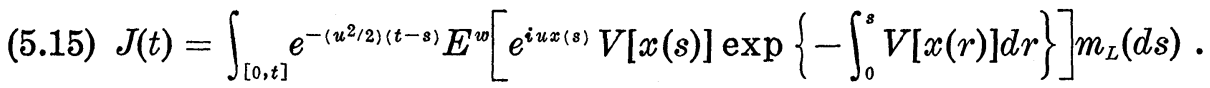

Applying Lemma 1 to the Wiener integral in (5.15) and recalling (5.1) and (5.3) we have

$$
\begin{aligned}
J(t) & =\int_{[0, t]} e^{-\left(u^{2 / 2)(t-s)}\right.}\left[\int_{R^{1}} e^{i u \eta} V(\eta) E\left(Y_{s} \mid X_{s}\right)(\eta) P_{X_{s}}(d \eta)\right] m_{L}(d s) \\
& =\int_{[0, t]} e^{-\left(u^{2 / 2)(t-s)}\right.}\left[\int_{R^{1}} e^{i u \eta} V(\eta) U(\eta, s) m_{L}(d \eta)\right] m_{L}(d s)
\end{aligned}
$$

Let us use (5.16) in (5.11). To interchange the order of the three integrals with respect to $m_{L}(d \eta)$ over $R^{1}, m_{L}(d s)$ over $[0, t]$ and $m_{L}(d u)$ over $(-a, a)$ in the resulting expression for $I_{2}$, observe that

$$
\begin{aligned}
&\left|\left(1-\frac{|u|}{a}\right) e^{-i u \xi} e^{i u \eta} V(\eta) U(\eta, s) e^{-\left(u^{2} / 2\right)(t-s)}\right| \leqq V(\eta) U(\eta, s) e^{-\left(u^{2} / 2\right)(t-s)} \\
& \text { for } \quad(\eta, s, u) \in R^{1} \times[0, t] \times(-a, a) .
\end{aligned}
$$

Recalling (5.3) and Lemma 1 we have by (2.4) and (5.5)

$$
\begin{aligned}
\int_{R^{1}} V(\eta) U(\eta, s) m_{L}(d \eta) & =E^{w}\left[V[x(s)] \exp \left\{-\int_{0}^{s} V[x(r)] d r\right\}\right] \\
\leqq E^{w}[V[x(s)]] & =\frac{1}{\sqrt{2 \pi s}} \int_{R^{1}} V(\zeta) e^{-\zeta^{2 /(2 s)}} m_{L}(d \zeta)=\varphi(s) .
\end{aligned}
$$

Now

$$
\int_{[0, t]} \varphi(s) e^{-\left(u^{2} / 2\right)(t-s)} m_{L}(d s)=A e^{-u^{2} t / 2}
$$

where

$$
A=\int_{[0, t]} \varphi(s) e^{u^{2} / 2} m_{L}(d s)<\infty
$$

by the continuity of $\varphi$ on $[0, \infty)$ and thus 


$$
\int_{R^{1}} A e^{-u^{2} t / 2} m_{L}(d u)<\infty
$$

This verifies the applicability of the Fubini theorem in interchanging the order of the three integrals. Thus we have

$$
\begin{aligned}
I_{2}(\xi, t)= & \lim _{a \rightarrow \infty} \frac{1}{2 \pi} \int_{[0, t]}\left\{\int _ { R ^ { 1 } } V ( \eta ) U ( \eta , s ) \left[\int_{R^{1}} \chi_{(-a, a)}(u)\left(1-\frac{|u|}{a}\right)\right.\right. \\
& \left.\left.\times e^{-i u(\xi-\eta)} e^{-\left(u^{2} / 2\right)(t-s)} m_{L}(d u)\right] m_{L}(d \eta)\right\} m_{L}(d s) .
\end{aligned}
$$

For all $a>0$ the integrand in (5.17) is bounded by

$$
V(\eta) U(\eta, s) e^{-\left(u^{2 / 2)(t-s)}\right.} \quad \text { for } \quad(\eta, s, u) \in R^{1} \times[0, t] \times R^{1}
$$

which is integrable with respect to $\left(m_{L} \times m_{L} \times m_{L}\right)(d(\eta, s, u))$ on $R^{1} \times$ $[0, t] \times R^{1}$ as we saw above. Thus by the Dominated Convergence Theorem

$$
\begin{aligned}
I_{2}(\xi, t)= & \frac{1}{2 \pi} \int_{[0, t]}\left\{\int_{R^{1}} V(\eta) U(\eta, s)\right. \\
& \left.\times\left[\int_{R^{1}} e^{-i u(\xi-\eta)} e^{-\left(u^{2} / 2\right)(t-s)} m_{L}(d u)\right] m_{L}(d \eta)\right\} m_{L}(d s) \\
= & \int_{[0, t]}\left\{\int_{R^{1}} V(\eta) U(\eta, s) \frac{1}{\sqrt{2 \pi(t-s)}} e^{-(\xi-\eta)^{2} /(2(t-s))} m_{L}(d \eta)\right\} m_{L}(d s) .
\end{aligned}
$$

Using (5.13) and (5.18) in (5.9) we have (5.4).

\section{REFERENCES}

1. R. R. Goldberg, Fourier Transforms, Cambridge University Press, 1961.

2. M. Kac, Probability and Related Topics in Physical Sciences, Interscience Publishers, New York, 1959.

3. R. E. A. C. Paley, N. Wiener and A. Zygmund, Notes on random functions, Mathematische Zeitschrift, 37 (1933), 647-668.

4. J. Yeh, Inversion of conditional expectations, Pacific J. Math., 52 (1974), 631-640.

Received May 12, 1975.

UNIVERsity of CALifornia, Irvine 


\title{
PACIFIC JOURNAL OF MATHEMATICS
}

\section{EDITORS}

RichaRd ARENS (Managing Editor)

University of California

Los Angeles, California 90024

\section{R. A. Beaumont}

University of Washington

Seattle, Washington 98105
J. DUGUNDJI

Department of Mathematics University of Southern California Los Angeles, California 90007

D. Gilbarg and J. Milgram

Stanford University

Stanford, California 94305

\section{ASSOCIATE EDITORS}
E. F. BECKENBACH
B. H. NeUmanN
F. WOLF
K. YoshIDA

\section{SUPPORTING INSTITUTIONS}

\author{
UNIVERSITY OF BRITISH COLUMBIA \\ CALIFORNIA INSTITUTE OF TECHNOLOGY \\ UNIVERSITY OF CALIFORNIA \\ MONTANA STATE UNIVERSITY \\ UNIVERSITY OF NEVADA \\ NEW MEXICO STATE UNIVERSITY \\ OREGON STATE UNIVERSITY \\ UNIVERSITY OF OREGON \\ OSAKA UNIVERSITY
}

\author{
UNIVERSITY OF SOUTHERN CALIFORNIA \\ STANFORD UNIVERSITY \\ UNIVERSITY OF TOKYO \\ UNIVERSITY OF UTAH \\ WASHINGTON STATE UNIVERSITY \\ UNIVERSITY OF WASHINGTON \\ AMERICAN MATHEMATICAL SOCIETY
}

The Supporting Institutions listed above contribute to the cost of publication of this Journal, but they are not owners or publishers and have no responsibility for its content or policies.

Mathematical papers intended for publication in the Pacific Journal of Mathematics should be in typed form or offset-reproduced, (not dittoed), double spaced with large margins. Underline Greek letters in red, German in green, and script in blue. The first paragraph or two must be capable of being used separately as a synopsis of the entire paper. Items of the bibliography should not be cited there unless absolutely necessary, in which case they must be identified by author and Journal, rather than by item number. Manuscripts, in triplicate, may be sent to any one of the editors. Please classify according to the scheme of Math. Reviews, Index to Vol. 39. All other communications should be addressed to the managing editor, or Elaine Barth, University of California, Los Angeles, California, 90024.

The Pacific Journal of Mathematics expects the author's institution to pay page charges, and reserves the right to delay publication for nonpayment of charges in case of financial emergency.

100 reprints are provided free for each article, only if page charges have been substantially paid. Additional copies may be obtained at cost in multiples of 50 .

The Pacific Journal of Mathematics is issued monthly as of January 1966. Regular subscription rate: $\$ 72.00$ a year (6 Vols., 12 issues). Special rate: $\$ 36.00$ a year to individual members of supporting institutions.

Subscriptions, orders for back numbers, and changes of address should be sent to Pacific Journal of Mathematics, 103 Highland Boulevard, Berkeley, California, 94708.

PUBLISHED BY PACIFIC JOURNAL OF MATHEMATICS, A NON-PROFIT CORPORATION

Printed at Kokusai Bunken Insatsusha (International Academic Printing Co., Ltd.), 8-8, 3-chome, Takadanobaba, Shinjuku-ku, Tokyo 160, Japan.

Copyright (C) 1975 by Pacific Journal of Mathematics Manufactured and first issued in Japan 


\section{Pacific Journal of Mathematics}

\section{Vol. 59, No. $2 \quad$ June, 1975}

Aharon Atzmon, A moment problem for positive measures on the unit disc ........

Peter W. Bates and Grant Bernard Gustafson, Green's function inequalities for

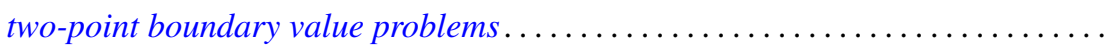

Howard Edwin Bell, Infinite subrings of infinite rings and near-rings ...........

Grahame Bennett, Victor Wayne Goodman and Charles Michael Newman, Norms of

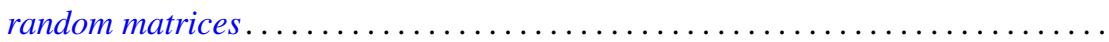

Beverly L. Brechner, Almost periodic homeomorphisms of $E^{2}$ are periodic.........

Beverly L. Brechner and R. Daniel Mauldin, Homeomorphisms of the plane ........

Jia-Arng Chao, Lusin area functions on local fields ......................

Frank Rimi DeMeyer, The Brauer group of polynomial rings ...............

M. V. Deshpande, Collectively compact sets and the ergodic theory of

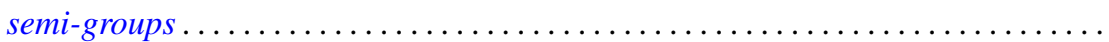

Raymond Frank Dickman and Jack Ray Porter, $\theta$-closed subsets of Hausdorff

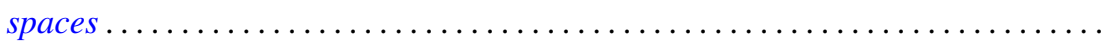

Charles P. Downey, Classification of singular integrals over a local field .......... 407

Daniel Reuven Farkas, Miscellany on Bieberbach group algebras . . . . . . . . . . . .

Peter A. Fowler, Infimum and domination principles in vector lattices . . . . . . . . . .

Barry J. Gardner, Some aspects of T-nilpotence. II: Lifting properties over

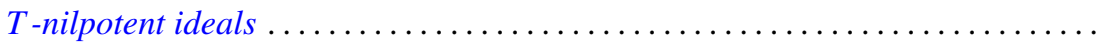

Gary Fred Gruenhage and Phillip Lee Zenor, Metrization of spaces with countable

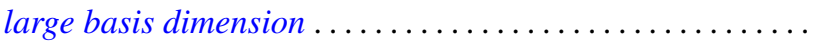

J. L. Hickman, Reducing series of ordinals...

Hugh M. Hilden, Generators for two groups related to the braid group ...

Tom (Roy Thomas Jr.) Jacob, Some matrix transformations on analytic sequence

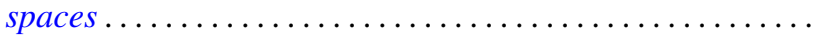

Elyahu Katz, Free products in the category of $k_{w}$-groups . .....

Tsang Hai Kuo, On conjugate Banach spaces with the Radon-Nikodým property...

Norman Eugene Liden, $K$-spaces, their antispaces and related mappings ...

Clinton M. Petty, Radon partitions in real linear spaces ........

Alan Saleski, A conditional entropy for the space of pseudo-Menger maps ....

Michael Singer, Elementary solutions of differential equations .

Eugene Spiegel and Allan Trojan, On semi-simple group algebras. I. . .

Charles Madison Stanton, Bounded analytic functions on a class of open Riemann

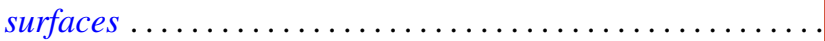

Sherman K. Stein, Transversals of Latin squares and their generalizations ....

Ivan Ernest Stux, Distribution of squarefree integers in non-linear sequences . . .

Lowell G. Sweet, On homogeneous algebras ................

Lowell G. Sweet, On doubly homogeneous algebras .......... .

Florian Vasilescu, The closed range modulus of operators ......

Arthur Anthony Yanushka, A characterization of the symplectic groups $\operatorname{PSp}(2 m, q)$

as rank 3 permutation groups... 